\title{
Langue et culture : l'indispensable association dans l'enseignement du FLE à des arabophones
}

\author{
Ilham Dupont \\ DITEC - Didactique des langues, des textes et des cultures \\ Université Sorbonne Nouvelle-Paris III \\ ilham.dupont@psuad.ac.ae \\ Omar Colombo \\ CSTI - Cultures, sociétés et technologies de l'information \\ Université Sorbonne-Paris IV \\ $\&$ \\ LIDILEM - Laboratoire de Linguistique et Didactique des Langues Etrangères et Maternelles \\ Université Stendhal-Grenoble III \\ omar.colombo@psuad.ac.ae; omcolombo@yahoo.fr
}

\section{Une problématique autour du plurilinguisme et de la pluriculturalité}

Nous enseignons respectivement le français et l'italien en tant que langues étrangères depuis des années. Cet enseignement, nous l'avons pratiqué dans différentes conditions et avec des apprenants de populations, de niveaux ou d'origines très diverses. S'il y a un premier constat que nous avons pu tirer de cette expérience, c'est qu'au-delà d'une pédagogie que l'on pourrait qualifier de "classique », il est incontournable de tenir compte de la situation linguistique et culturelle de l'apprenant. Langue et culture sont intimement liées et l'acquisition de l'une ne peut se faire sans la compréhension de l'autre.

Le deuxième constat auquel nous avons été amenés est que, quelques soient les conditions d'enseignement, on retrouve toujours le même détecteur commun des failles et des progrès de l'apprenant : ce sont les erreurs. Comment les identifier et les qualifier constitue notre approche.

La problématique comporte deux axes de recherches principaux :

Axe 1 : Acquisition et apprentissage : L'autocontrôle et la métacognition, figurent-elles parmi les facteurs et les aspects qui accompagnent l'apprentissage de L2 ? Les apprenants font-ils une réflexion métalinguistique approfondie pour le choix lexical ? La proximité interlinguistique est-elle un enjeu crucial parmi les procédés psycholinguistiques réalisés dans l'apprentissage lexical de L2 ?

Axe 2 : Culture/lexiculture, nativisation/dénativisation: Les apprenants sont-ils influencés par leur culture maternelle? Comment s'exerce-t-elle? L'interlangue est une phase nécessaire entre nativisation et dénativisation? La lexiculture est-elle une solution efficace dans l'apprentissage de L2?

Les hypothèses suivantes sont associées à cette problématique : la culture maternelle perturberait l'apprentissage de L2 ; la culture de L2 devrait être associée à celle la langue. L2 et sa culture seraient comme les deux faces d'une même pièce. Il y aurait des transferts interlinguistiques dans la production écrite en français.

Notre expérience actuelle dans le cadre de l'Université Paris-Sorbonne Abou Dhabi (PSUAD) aux Émirats Arabes Unis (EAU) où nous enseignons le français et l'italien à des populations hétérogènes (soixante-cinq nationalités) nous procure de nouveaux paramètres d'investigation. Ce contexte nous a renforcé dans nos convictions que seule une analyse exhaustive des erreurs dans l'écrit et la qualification de leurs sources est le point de départ pour faire progresser l'apprentissage de L2 (ou plus) par des apprenants étrangers. L'analyse des erreurs ne doit évidemment pas être une fin en soi et nous ne la proposons pas comme telle. Elle est le point de départ d'une nécessaire évolution dans l'enseignement d'une langue et de la culture qui y est associée. 
La conclusion de ce travail sur l'analyse contrastive des productions en français de locuteurs arabophones, montrera comment à partir d'un constat analytique de leurs erreurs, on peut amener les apprenants à se trouver dans une démarche réfléchie, subjective, interactive et productive.

L'analyse dont nous nous servons a été faite dans un contexte arabophone car dans notre expérience, les étudiants arabes sont majoritaires. L1, dans ce cas, la langue arabe qui est très éloignée du français et de l'italien L2, tant par l'oral que par l'écrit, renforce l'exemplarité de notre démonstration. Les constats que nous proposons sont applicables aussi bien à l'anglais ou à d'autres langues.

\section{Présentation du cadre géolinguistique, institutionnel et pédagogique}

L'arabe est une langue sémitique comme l'hébreu ou l'araméen. En fait, deux types d'arabe coexistent, l'arabe dialectal et l'arabe littéraire.

Il y a en arabe, de nombreuses variétés dialectales en fonction des pays et même des régions géographiques. Ce sont ces très nombreux dialectes qui sont les plus usités. Ils constituent les langues de communication et de transmission de la culture et des traditions. À la fois différents et proches, ils permettent une intercompréhension dans l'ensemble du monde arabe bien que certains mots puissent avoir des sens très différents.

L'arabe littéral est enseigné dès le primaire dans les écoles de l'ensemble du monde arabe puisqu'elle est la langue du coran, de la littérature et de la culture. Elle y est donc la plus répandue. Il y a des similitudes linguistiques entre l'arabe littéraire et les dialectes mais de fait, l'arabe littéraire reste la langue des intellectuels.

Les Émirats Arabes Unis se situent au sud-ouest de l'Asie, dans la péninsule arabique. En 2006, la population atteignait environ 5 millions et demi d'habitants dont la majorité est constituée d'immigrés. Aux EAU, la langue officielle est l'arabe mais l'anglais étant la langue commune, est très largement utilisé en particulier dans les milieux d'affaires. Les documents officiels doivent être obligatoirement rédigés en arabe mais pour des raisons évidentes, ils sont fréquemment bilingues avec l'anglais. On peut noter une certaine dévalorisation du dialecte par la société émirienne au profit de l'anglais. En matière d'éducation, l'enseignement est prodigué en arabe et aussi en anglais, ce qui est obligatoire depuis 1985.

L'ouverture de l'Université Paris-Sorbonne Abou Dhabi en 2006, a permis d'introduire l'enseignement en français et la présence de la culture française aux Émirats. L'université est ouverte à tous les étudiants (autour de soixante-cinq nationalités représentées). Elle propose le niveau de la Licence et du Master, en introduisant progressivement les principales disciplines enseignées à la Sorbonne de Paris. Les cours de licence sont assurés en français et étant donné que la quasi-totalité des étudiants qui s'inscrivent à l'université, n'ont pas ou très peu connaissance de la langue, c'est le rôle du département de Français Langue Etrangère de les amener dans les meilleurs délais à une maîtrise suffisante pour être capables de suivre ces cours dans des conditions universitaires normales (comparables à celles de la France). L'obtention du diplôme B1-CECR (quatrième niveau), un diplôme délivré par PSUAD sous le nom de Diplôme d'Université, permet de s'inscrire en licence dans l'un des départements.

\section{Encadrement scientifique : VALICO et la pertinence de l'image en classe de langue}

\subsection{Présentation du projet VALICO}

VALICO est l'acronyme de Varietà di Apprendimento della Lingua Italiana : Corpus Online, un corpus de productions écrites d'apprenants étrangers à l'italien LE, accessible on line (lien : http://www.corpora.unito.it/) $)^{1}$. Il s'agit d'un corpus de productions écrites d'apprenants étrangers, qui peut devenir un outil didactique et scientifique à disposition des enseignants, des étudiants universitaires et des chercheurs dans ce domaine. De ce point de vue, VALICO permet de sélectionner les écrits des apprenants d'après plusieurs paramètres (la LM, les L2 connues, l'âge et le niveau d'étude); il permet également d'examiner l'ensemble des productions d'un même étudiant ou d'une même classe de langue. 
L'équipe a ainsi créé des dessins narratifs et descriptifs à partir desquels les apprenants doivent constituer leur production écrite, ou leur ekphrasis (Eco, 2006). Les dessins narratifs qui nous concernent ici sont composés de quatre ou de six vignettes, l'étudiant doit s'en inspirer pour écrire une histoire. Chaque dessin est composé d'une consigne et d'un incipit (ou amorce) qui servent de guide pour l'étudiant. Avant le début de l'expérience, chaque étudiant doit remplir un Questionnaire auteur dont l'objectif est de définir le profil du rédacteur. De plus, le questionnaire interroge le rédacteur sur ses connaissances possibles de L2, de communication autre que la LM, sur le milieu plurilingue, l'anglais dans les Émirats Arabes Unis, ainsi que le français, l'italien, l'espagnol, l'allemand, etc. Nous avons décidé d'adapter l'un des dessins de VALICO conçu pour l'italien L2 pour rejoindre nos objectifs de recherche : obtenir et analyser des textes produits en FLE. En effet, les images VALICO démarquent non seulement de l'intérêt scientifique, mais également de la pertinence pédagogique en classe de langue.

\subsection{La pertinence de l'image en classe de langue}

Depuis plusieurs années l'image a pris sa place parmi les méthodologies de l'enseignement / apprentissage d'une L2. Le rôle de l'illustration a connu une évolution conceptuelle au fil du temps, néanmoins «il n'est plus possible à l'école d'ignorer l'image [...]» (Mallet, 1989: 120) comme un adjuvant à la leçon de langue. Ainsi, notre propos est ici de justifier brièvement le choix du support iconographique dans l'enquête en exposant les fonctions de l'image en classe de langue.

L'introduction des nouvelles technologies en classe de langue a permis d'exploiter des images à la fois ludiques (l'image doit plaire, séduire et captiver - Tardy, 1975:29) et fictives (l'image n'est pas la réalité, mais un signe de ce réel - Mallet, 1989: 124). L'utilisation de l'image est une approche constructive lorsque le formateur demande aux apprenants «de réaliser des énoncés sémantiquement équivalents, de tenter des paraphrases » (Coste, $1975: 13$ ) afin que l'étudiant soit amené à effectuer des efforts métalinguistiques en explicitant le sens littéral du texte ou en proposant des variantes sémanticosyntaxiques.

Les représentations dans le lexique mental se forment par des procédés d'analyse, de codification et de reconnaissance qui élaborent l'input sur la base des caractéristiques spécifiques avec lesquelles il se présente ; ainsi, il est très important dans l'apprentissage d'une L2 de présenter l'input linguistique à travers le nombre le plus élevé possible de codes linguistiques (Cardona, $2004: 25)$, donc également par le code visuel ; en effet, avec l'action de plusieurs processus de codification on augmente les probabilités de parvenir à une représentation stable dans le lexique mental (ibidem).

\section{Méthodologie de l'enquête}

Le dessin que nous avons choisi pour cette enquête, titrée LOVE, est composé de quatre vignettes ${ }^{2}$, à partir desquelles les étudiants doivent rédiger un texte, selon les consignes suivantes : narrer une histoire, éviter la description, le texte doit comporter au moins 100 mots et commencer par l'amorce «Ieri al parco... », la traduction française a été écrite au tableau («Hier, au jardin public... »).

Les apprenants qui ont participé à cette expérience : 10 étudiants inscrits en $2^{\text {ème }}$ ou en $3^{\text {ème }}$ année de Licence en Langues Étrangères Appliquées (LEA) ${ }^{3}$. L'âge des apprenants varie de 20 à 25 ans. Tous les étudiants étaient arabophones. Dans le cadre de LEA, les apprenants devaient choisir entre deux langues principales : certains ont opté pour l'arabe, d'autres pour l'anglais. Pour tous, l'italien était la $2^{\text {ème }}$ langue de spécialité. Toutefois, dans leur cursus universitaire, les étudiants sont obligés de continuer leur formation en FLE. En ce qui concerne l'apprentissage de l'italien, les groupes présentent des niveaux mixtes dans cette langue, de A2 à B2 (CECR), l'ayant étudié au moins pendant 2 ans. Le contact avec la langue cible (LC) et l'italien ne se limite pas uniquement à l'université : les apprenants ont fait des voyages en France et Italie (séjours linguistiques, vacances, parfois même des stages professionnels) et ils sont souvent en contact avec ces deux langues au moyen des mass media (radio, TV, Internet), parfois grâce à des amis français et/ou italiens. D'autres L3 connues sont soit l'allemand, soit l'espagnol. 
Le jour de l'expérience, nous avons expliqué aux étudiants les modalités du traitement des données contenues dans le Questionnaire auteur (qui a été remplis en $10 \mathrm{mn}$ ), précisément l'obtention de leur autorisation pour l'exploitation de leur production écrite. Les étudiants savaient qu'ils devaient rédiger leur texte de manière autonome, sans avoir la possibilité de questionner l'enseignant ni de consulter d'autres documents et ils ont montré leur volonté de s'engager dans l'expérience. Ils ont avoué avoir éprouvé des difficultés lexico-sémantiques et syntaxiques mais ont trouvé, cependant, la tâche narrative accessible.

\section{L'importance du lexique et de la lexiculture dans l'apprentissage d'une L2}

\subsection{Le lexique}

Étudier une L2 ne se limite pas à l'apprentissage cumulatif et étanche des règles syntaxiques, des mots et de la grammaire sans liens avec des objectifs précis. L'approche communicative nous enseigne que l'apprentissage d'une L2 doit permettre à l'étudiant de communiquer quelque chose en situation d'échange. L'approche actionnelle ajoute que l'apprenant est un «acteur social dont le rôle est d'accomplir des tâches [...] dans des circonstances et un environnement donnés et dans un domaine particulier» (CECR, 2001: 15). Ainsi, parmi les besoins d'un étudiant, le CECR pointe les besoins langagiers, qui correspondent aux exigences de faire quelque chose avec une L2 dans une situation précise. Parmi les compétences que l'apprenant doit acquérir pour accomplir une tâche spécifique, il y a les compétences linguistiques; notamment, la compétence lexicale qui, d'après Ferreri (2005: 309), est la plus importante, bien que le lexique ait été ignoré pendant longtemps par la linguistique appliquée: " vocabulary acquisition is part of the psychology of second language learning that has received short shrift from applied linguistics, and has been very largely neglected by recent developments in research » (Meara, 1980 : 121). De même, dans le cadre du FLE, d'après Duquette \& Tréville (1996: 7) le vocabulaire «est rarement au centre des méthodologies dites communicatives ». La position centrale du lexique en L2 est ouvertement évoquée par l'approche communicative : Widdowson (1978) remarquait qu'une phrase agrammaticale mais correcte lexicalement est plus facile à comprendre qu'une phrase acceptable grammaticalement mais incompréhensible pour son lexique. Avec le lexique l'apprenant pourra désigner les objets du monde, concrets ou abstraits, proches ou éloignés dans le temps et dans l'espace. Ainsi, le lexique devient particulièrement important dans l'apprentissage et la didactique d'une L2 : l'appropriation progressive du lexique le plus fréquent d'une langue donnée permet à l'apprenant de disposer des instruments indispensables dans les échanges langagiers en LC.

\subsection{La lexiculture}

De nombreuses erreurs, pour beaucoup lexicales, ont pour cause identifiable la méconnaissance du contexte de la langue L2. Les étudiants se réfèrent à ce qu'ils apprennent, syntaxe, grammaire, lexique, etc., et nous verrons avec la nativisation, comment l'apprenant compense ses lacunes par des références à sa propre langue L1 et produit ainsi des erreurs.

Pendant longtemps, l'enseignement s'est cantonné à l'apprentissage didactique de la langue. Bien que certains voyaient l'intérêt d'enseigner la culture en parallèle, celle-ci est restée dissociée de celle de la langue. Reprenant une idée déjà exprimée, Galisson a présenté en 1986 un concept qui a fait l'effet d'une révolution dans l'enseignement des langues étrangères en montrant que langue et culture ne pouvaient être dissociées et que leur enseignement devait être simultané. Il a montré que c'était le meilleur moyen pour aboutir à ce qu'un apprenant puisse utiliser la langue L2 dans sa culture propre, ce qui est la finalité de l'apprentissage effectif d'une langue étrangère.

Le concept de la «lexiculture »: le terme lexiculture est une contraction de lexique et de culture car le concept est basé sur l'utilisation d'un lexique comportant des «mots culturels » ou qui font référence à la culture. 
Le lexique : c'est un recueil de mots qui ont un signifiant et un signifié. L'idée est qu'au-delà du sens que peut fournir un dictionnaire, des mots ou des unités lexicales ont une signification culturelle, une dimension socioculturelle, parfois sous-jacente ou induite. Un sens que comprend naturellement les natifs de la langue mais qui pose problème pour son apprenant. On comprend pourquoi les mots, dont la signification habituelle apparait insuffisante, doivent être expliqués dans tous les sens qu'ils comportent, autant sur le plan lexical que sur celui culturel qui lui est attaché. Les expressions pragmaticosémantiques, une fois repérées en fonction de la langue, doivent être répertoriées et organisées, pour constituer un lexique des unités lexicales « lexiculturelles ».

La culture : comment le mot culture qui englobe tant d'aspects, est compris dans la lexiculture ? La culture est prise ici dans son ensemble et dans tous ses aspects. Ceux qui décrivent la vie quotidienne partagée par la plupart des gens qui la pratiquent et ceux qui font référence à la vie de la pensée, à la vie cultivée, artistique ou institutionnelle. Pour les apprenants, il y a une différence sensible entre ces deux axes de la culture mais l'apprentissage de la culture doit être une combinaison des deux. La culture quotidienne est indispensable pour la communication avec les natifs mais elle est plus longue à comprendre et acquérir car pour les pratiquants, elle est intégrée au fil du temps depuis le plus jeune âge. Par contre, si elle est moins utile pour pratiquer la langue, la culture " intellectuelle » est disponible grâce à la littérature et aux medias, donc enseignable :

\section{«la spécificité de cette discipline est de proposer une entrée dans la culture par les mots, d'intégrer culture et langue dans une approche qui ne les disjoigne pas, qui respecte également leur intime consubstantialité, de privilégier la culture courante (donc la culture-action) sur la culture savante et d'accorder une grande importance à une culture lexicaliste de l'interculturel (...). »(Puren \& Galisson, 1999; cité par Véronique, $2007:$ 123).}

L'apprentissage de L2 comporte une part de connaissances techniques qui va permettre de comprendre et de s'exprimer dans la langue et, grâce à la «lexiculture», une dimension culturelle qui permet de communiquer et de comprendre des représentations subjectives que comprennent et partagent les natifs de la LC. En effet, une simple conversation entre deux personnes ayant la même culture peut être incompréhensible ou même inaccessible à un étranger. La pratique de l'enseignement de la culture simultanément à celle de la langue amène les apprenants à comprendre et interpréter dans une démarche réfléchie, subjective et interactive. Dans cette démarche, l'apprenant gagne en motivation à apprendre car il travaille dans le concret. À cela s'ajoute une autre dimension qui vient de l'enrichissement de l'apprenant par le contact entre les deux cultures, comme par un effet de miroir.

On imagine facilement sur ce plan, les difficultés que pose l'enseignement dans le cadre de l'Université Paris-Sorbonne Abou Dhabi avec autant de nationalités et de cultures différentes! Voici quelques exemples des interférences qui existent entre une langue et sa culture. Quelle peut être la compréhension d'un apprenant arabophone de L2 qui entend ou trouve dans un texte l'expression " la messe est dite » (dans le sens : tout est dit ou fait, il n’y a plus rien à dire ou à faire) ? Même le recours au dictionnaire ne peut lui fournir une réponse complète sur le mot «messe » et ce qu'il contient. Il ne suffira pas de lui donner une équivalence lexicale pour qu'il comprenne le sens de cette expression. Elle ne peut pas avoir de sens si elle n'est pas expliquée à l'apprenant par la culture qui est associée à la langue qu'il apprend. Cet exemple fort montre clairement à quel point la langue et la culture sont indissociables dans l'enseignement.

Voici un exemple d'erreur qui pourrait être prise pour courante ou commune. Dans la démarche que nous décrivons, on constate que cette erreur a pour source une interférence entre les cultures, en tous cas une méconnaissance de la culture associée à L2. Dans un récit, les apprenants doivent utiliser le terme femme ou fille. Dans le cas présent ce serait le terme femme qui serait le plus approprié, mais les apprenants utilisent les deux de manière « anarchique ». Dans la culture associée à L2, le clivage entre fille et femme est surtout lié à l'appréciation : âge apparent, catégorie sociale, mariée ou non, etc. Pour les natifs de L1, c'est la situation maritale qui fait la différence : pas mariée, c'est une fille; mariée, c'est une femme. C'est un exemple typique d'un locuteur qui n'ayant pas connaissance des pratiques culturelles associées à L2 et ne sachant pas quel est le bon terme à employer, utilisera l'un ou l'autre, ou se référera à sa propre 
«culture quotidienne ». L'utilisation des termes garçon et homme correspond d'ailleurs aux mêmes données culturelles.

Dans un autre récit, l'expression que l'on utiliserait en L2 serait «je ne veux plus voir ta tête ». Plusieurs apprenants ont utilisé le terme visage, «je ne veux plus voir ton visage ». Pourquoi ce choix qui pourrait passer pour une simple question de lexique ? Encore une fois, la réponse est dans l'analyse des erreurs. Ici cependant, l'erreur peut être considérée comme ayant pour source la nativisation mais aussi la culture. Si on se réfère à la culture de $\mathrm{L} 1$, on s'aperçoit que le visage a une place et une importance différente et il est beaucoup mentionné dans le Coran. Son sens n'est donc pas tout à fait le même que dans L2. Une différence qui, étant prise en compte par l'intégration à l'enseignement de la culture associée à la langue, évitera des incompréhensions et des erreurs tandis qu'il facilitera la communication.

\title{
5.3 Nativisation et dénativisation, Interlangue
}

$\mathrm{Au}$ cours de l'acquisition d'une langue étrangère L2, l'apprenant est tenté de se référer, faute d'informations suffisantes, à ce qu'il connait, c'est-à-dire à sa langue L1 et à son environnement. Deux types de connaissances, implicites et explicites, cohabitent dans son esprit et il utilise d'une part des schémas ou normes externes, et d'autre part ses connaissances personnelles. C'est une période de «nativisation» (Andersen, 1983) pendant laquelle cette dualité le fera produire des erreurs. L'apprenant établi toujours une relation entre les nouvelles données et celles qu'il a déjà en tête, au moyen d'une analyse contrastive effectuée en profondeur (voir point 6.1). Il fait des hypothèses sur le fonctionnement de la langue cible et utilise ses connaissances propres à sa langue source pour pallier celles qui lui font défaut. Il adapte ce qu'il apprend à ce qu'il sait plutôt que l'inverse :

\begin{abstract}
«L'apprentissage incident sera également et logiquement perturbé par des phénomènes de nativisation. Pour que la dénativisation soit efficace, un travail de vérification de ce qui est compris et produit, et donc une médiation, sera d'autant plus nécessaire que l'apprenant a des objectifs élevés (mais le médiateur ne saurait pouvoir tout "vérifier"). » (Demaizière \& Narcy-Combes, 2005).
\end{abstract}

Pour acquérir un nouveau savoir, il est nécessaire de prendre de la distance par rapport à ce que l'on sait. C'est ce que l'on fait naturellement plus ou moins vite dans l'apprentissage et l'apprenant va progressivement remplacer les références à L1 par celles liées à L2 jusqu'à ce qu'il arrive à ne plus se référer à sa langue source. C'est un travail de «dénativisation» dans lequel il doit s'impliquer personnellement. Dans le passage de nativisation à dénativisation, il y a une période pendant laquelle l'apprenant doit «abandonner » L1 pour «acquérir » L2. Cette situation évolutive de l'acquisition entre les deux langues est appelée « interlangue » :

\begin{abstract}
«Les modifications de l'interlangue de l'apprenant sont déclenchées par l'accumulation de constructions d'énoncés à partir de règles ou d'instances $[\ldots]$ accompagnée de la prise de conscience des écarts entre l'organisation de l'interlangue et celle de l'input, en prenant en compte le contexte de l'énonciation et l'intention de l'énonciateur. 》 (Demaizière \& Narcy-Combes, 2005).
\end{abstract}

Son mécanisme est donc variable en fonction du rapport L1/L2 et aussi, de l'apprenant lui-même qui doit faire un travail cognitif, une sorte de réapprentissage.

Les erreurs produites pendant cette phase peuvent être la conséquence de la confrontation entre les deux langues mais elles peuvent également être induites par la pédagogie elle-même. La pédagogie est principalement élaborée sur des hypothèses et en matière de production langagière, elle suit deux principales théories : un système basé sur des règles (Anderson, 1993) et un autre basé sur des modèles. Dans ce dernier, on utilise des phrases toutes faites ou modèles, qui servent en les calquant, de guide à la production de l'apprenant.

Particularités des systèmes langagiers et troubles phonologiques. Différents mécanismes s'imbriquent d'une manière complexe car deux systèmes fonctionnent parallèlement, ce qui provoque un important trouble phonologique entre les systèmes des deux langues. Le son que l'apprenant entend semble converti 
ou transcrit lorsqu'il arrive à son cerveau. Ce mécanisme s'effectue d'une manière inconsciente envers tous les sons ou structures qui sont étrangers à L1. De même, lorsqu'ils s'expriment, les gens ne s'entendent pas parler. Il faut donc qu'il y ait quelque part un relais qui fait l'association entre l'oral et l'écrit. Manifestement, ce handicap s'ajoute à une opération intellectuelle déjà compliquée et un cognitivisme ardu et difficile. Polianov explique la notion de surdité phonologique comme suit :

«[...] même en percevant des mots (ou des phrases) d'une langue avec un système phonologique tout différent, nous sommes enclins à décomposer ces mots en des représentations phonologiques propres à notre langue maternelle. En entendant un mot inconnu étranger [...] nous tâchons d'y retrouver un complexe de nos représentations phonologiques, et de le décomposer en des phonèmes propres à notre langue maternelle, et même en conformité avec nos lois de groupement des phonèmes » (Polianov, $1931: 88-89$ ).

Les difficultés phonétiques et phonologiques réciproques. Les spécificités des langues conduisent incontestablement à des difficultés phonologiques. Ainsi donc, les arabes ont des difficultés à prononcer certains sons en français.

Conjonction phonologique consonantique. L'interférence, c'est le contact de langues, c'est l'utilisation d'éléments de L1 alors que l'on écrit en L2. Ce phénomène affecte le lexique, la morphologie, la phonétique, la phonologie, bref toute la structure de L2. Les apprenants écrivent par exemple ce qu'ils entendent. Le cerveau est incapable de faire la distinction et donc ne filtre pas. C'est opaque. Les productions des apprenants de PSUAD montrent bien qu'ils perçoivent et analysent toute nouvelle langagière en L2 selon des critères déjà en place.

Les occlusives bilabiales [p] et [b] et des fricatives [f] et [v] en sont un bon exemple. Frapper devient * fraber et neuve devient *neuf, quelles que soit leurs positions, ces consonnes sont systématiquement remplacées par [b] et par [f]. Ces réalisations illustrent la nativisation. Cette substitution du [p] et du [v] qui est d'ailleurs générale, vient du fait que ces deux consonnes n'existent pas dans le système consonantique de L1. [b] et [p] se distinguent uniquement par le voisement. Elles sont remplacées par des sons qui ont les mêmes caractéristiques articulatoire que [p] et [v] puisqu'elles sont proches de ce que l'apprenant entend, soit [b] et [f], une occlusive bilabiale et une fricative.

Au début de l'apprentissage de L2 pour nos étudiants arabophones à PSUAD, la voyelle [y] arrondie antérieure est systématiquement substituée par [u]. Par exemple, le mot «rue » est prononcé [ru], or ce mot existe aussi mais avec un autre sens. Il s'agit du processus de nativisation. Sur ce point, nous avons pu remarquer qu'après avoir fortement insisté sur ce phénomène vocalique et grâce à un travail cognitif sensible des apprenants, ils avaient réussi à s'autocontrôler et à éliminer l'erreur dans un processus de dénativisation.

C'est ce mélange des deux langues au sein de l'interlangue qui produit des erreurs typiques dont les sources sont reconnaissables. On constate, par exemple, que lorsqu'un apprenant veut parler de quelques coups portés par une personne sur une autre, il utilisera fréquemment le terme «quelques frappes ». On a affaire à un phénomène de nativisation car il s'agit d'un emprunt à L1 dans la production de L2. Le terme utilisé est la traduction de l'expression arabe.

Ce phénomène de nativisation peut encore être illustré par cet exemple. Dans le cours d'une production écrite, pour désigner une même chose, une personne par exemple, l'étudiant va faire appel alternativement à un genre ou l'autre en fonction du contexte de son récit. Cette erreur pourrait être attribuée à un manque d'attention ou à une hésitation pour le terme approprié. En fait, on constate dans une démarche analytique que l'utilisation du masculin ou du féminin correspond au rapprochement par le contexte de L1 ou de L2. Si la phrase est plus proche de L1, il utilisera le genre de L1 et de même pour L2. Dans les productions de nos étudiants on remarque bien qu'ils ont sélectionné des éléments qui leur apparaissent pertinents que ce soit sur le plan phonologique, morphologique, syntaxique ou lexical et qui ne sont pas forcément conforme aux normes de L2. La sélection qui varie d'un apprenant à l'autre, s'effectue de manière différente. Ces étudiants sont tombés dans des erreurs du fait que l'écrit est 
beaucoup plus abstrait que l'oral car il $\mathrm{n}$ y a ni son ni interlocuteur. Ils ont fait et doivent encore faire des efforts de volonté pour écrire et être conscients de ce qu'ils doivent produire, ce qui les conduit à faire des erreurs. Les étudiants apprennent en même temps l'écrit et l'oral, d'où les difficultés.

Les étudiants qui possèdent une bonne maîtrise du système linguistique et sont capables de s'exprimer oralement ne peuvent pour autant communiquer. À la maitrise de la langue, il leur manque la capacité à savoir s'en servir dans un contexte social déterminé. On pourrait dire qu'aux moyens techniques il faut ajouter le « savoir-faire » ou plus exactement dans ce cas, «le savoir dire ». Il s'agit d'une question plus générale car on retrouve les mêmes caractéristiques chez les étudiants et les élevés dont L1 est le français : il y a une confusion entre le langage oral et écrit, bien qu'il y ait un code qui les distingue. C'est l'écriture et l'orthographe qui permettent le passage de l'un à l'autre. L'objectif pour l'apprenant est de communiquer et de s'exprimer de façon intelligible conformément à ce qu'il veut dire. S'il y a des règles linguistiques communes à l'expression orale et écrite, il y a cependant des conditions propres à chacun de ces modes. La syntaxe intègre la dimension orthographique. L'acquisition de l'écrit montre bien le lien nécessaire mais non suffisant, entre la langue orale et la langue écrite. Pour illustrer certains effets de ces difficultés pour passer de l'oral à l'écrit, on a remarqué que dans les productions écrites qui mobilisent simultanément, la réflexion, l'imagination et l'écriture, on retrouve sur l'ensemble des copies, les mêmes caractéristiques rythmiques propres à un récit oral. On y identifie les mêmes coupures et tournures syntaxiques. Le texte est " haché », avec presque autant de phrases que de lignes et les phrases sont comme stéréotypées avec simplement un nouvel ingrédient à chacune. Même le choix lexical est proche de celui utilisé dans un récit oral.

L'écriture ne peut pas être dissociée du système linguistique. Lorsque l'on écrit, on pense aux idées et à la manière de les faire naitre mais aussi, et en même temps, à utiliser les règles grammaticales et orthographiques intériorisées dans le cerveau. Le cerveau est activement sollicité dans ce processus simultané. La communication est moins affinée dans l'expression orale par manque de temps alors que ce même temps qui manque, bénéficie à la qualité de l'expression écrite.

Parmi les erreurs dues aux interférences entre L2 et L1 se trouve le genre. Il n'y a pas de règle qui gère le genre en français. L'apprenant ne pourra donc savoir ou prévoir le genre en français et, dans ce cas, d'une manière inconsciente il fait recours à celui de L1. Exemple : personne ou météo qui sont respectivement masculin en L1, seront transposés comme telle en français (*le personne, *le météo). Dans L1 le féminin est différencié du masculin morphologiquement.

Le dédoublement du sujet ne peut être accepté en L2 que dans le cas d'une emphase (accent particulier porté sur un constituant de la phrase), ou de structures de dislocation en déplaçant le GN de sa place normale, en laissant derrière lui un pronom anaphorique pour le représenter.

Pour les apprenants de L2, le verbe est toujours accompagné en L1 de désinences ou de préfixes qui correspondent au genre, au nombre, à la personne et à l'aspect verbal et donc le pronom personnel est exprimé par la forme verbale. Le pronom est ainsi toujours associé dans le verbe. Certaines erreurs pourraient être parfois possibles ou plausibles phonologiquement comme dans *quiter / quitter, *fraper / frapper. Dans L2, il existe un certain nombre de consonnes géminées : les dentales, les fricatives, les nasales, les bilabiales et les liquides. Phonologiquement certaines consonnes géminées se prononcent différemment des consonnes simples. Le [s] de grosse par exemple, se réalise phonétiquement en [s], alors que dans onctuosité on entend le son [z]. Aussi, ils ont tendance à généraliser des règles grammaticales ( $t u$ *veus au lieu de veux ; ils les *manges), ils utilisent la deuxième personne du verbe.

L'orthographe grammaticale (complémentairement avec l'orthographe lexicale), qui consiste à mentionner les fonctions grammaticales et déterminer graphiquement les éléments variables, comme la marque du genre ou du nombre, la conjugaison des verbes, l'accord du participe passé, l'accord en nombre du nom, etc., comporte quantitativement un nombre important d'irrégularités puisque les marques morphologiques n'apparaissent pas oralement.

Parmi les difficultés particulières à L1, il y a le cas des verbes avoir et être. Ces deux verbes n'existent pas dans la langue arabe. Ils peuvent cependant être sous-entendus. Le verbe avoir peut être exprimé par la préposition chez suivie d'un suffixe. L'équivalent du verbe être, ce sont les pronoms personnels au 
présent. Au passé, on le remplace par le verbe «kaana ». Cette situation créé chez les apprenants de L2 une grande confusion. Ils ne savent même pas quel auxiliaire employer pour un temps composé. À titre d'exemple : «cet homme *a tombé amoureux », « il *avait bagarré », «l'histoire *a fini ».

\subsection{Le rôle de l'Interlangue en français}

Nous avons relevé plusieurs erreurs de nature graphophonologique et morphosémantique.

Il est souvent difficile pour un apprenant de détecter le positionnement des accents graphiques, surtout lorsque la représentation des correspondances graphie/phonie se situe dans un niveau peu développé d'interlangue en LC (niveau initiale/intermédiaire) : *dériere pour derrière, *proteger pour protéger, *enervée pour énervée, *repond pour répond, etc.

La similitude formelle ou de signifiants (Corda \& Marello, 2004 : 21) amène souvent les étudiants à associer, en production, une forme et une signification qui ne lui correspond pas, sur la base des ressemblances formelles (Calaque, 2000 : 22). Par exemple, l'erreur *tros pour trop est probablement due à une confusion entre les mots trop et très, les deux étant des monosyllabes caractérisés par la même attaque consonantique. Plus généralement, deux ou plusieurs mots pourraient conduire l'étudiant à une erreur d'encodage lorsqu'ils sont similaires, non homographes mais homophones ou phonétiquement très proches : *marie pour mari, j'étais en *trein de pour j'étais en train de, etc.

Les ressemblances linguistiques peuvent contribuer à développer des représentations cognitives singulières et erronées autour des correspondances graphophonologiques de la LC : *famme pour femme, * problem pour problème, le *privet pour le privé (au sens de « la vie privée »), etc.

Souvent, les apprenants ont effectué une substitution paradigmatique transcatégorielle (*arrêt $\mathrm{N}$ pour arrête $\mathrm{V}$, etc.), ou dans la même catégorie syntaxique, principalement à l'intérieur de la catégorie verbale (on * veux pour on veut, il a $\underline{\text { vois }}$ pour il a $\underline{\mathrm{vu}}$, etc.) et nominale (*marie pour mari, il *a porté sur la * main pour il l'a portée sur le dos/les épaules, etc.). La substitution nominale est due à une confusion lexicale entre hyponymes (dans nos exemples, *mains pour épaules) qui appartiennent au même champ sémantique. Petiot \& Reboul-Touré (2005 : 219) désigneraient ces phénomènes d'échanges linguistiques en LC comme des substituts prévisibles en langue : à propos des substitutions d'un hyponyme avec son hyperonyme (ou, nous pouvons ajouter, par un co-hyponyme), les auteurs affirment qu'elles «sont prévisibles dans la mesure où l'ensemble de leurs traits sémantiques est compris dans la définition du nom retenu » (ibidem). Notons également que parfois un effet d'amorçage ou de priming pourrait se réaliser : il est question ici de l'activation d'un champ sémantique sous l'effet de la présentation d'un item lexical qui facilite le traitement cognitif d'autres items appartenant au même champ sémantique (Coirier \& al., $1996: 155$, note 2). Si l'objet extralinguistique, visualisé sur l'image, est considéré comme ambigu ou si le mot en LC est inconnu, le rédacteur opte pour la mention de l'élément sémantiquement superordonné (hyperonyme) ou sémantiquement proche (co-hyponyme), probablement en conséquence de la visualisation d'autres objets dans les vignettes, d'autres co-hyponymes de l'objet cible. En ce sens, l'appui sur le contexte situationnel de l'image peut se révéler déterminant.

\section{D'autres aspects cognitifs et psycholinguistiques dans l'encodage lexical}

De nombreuses études effectuées par la linguistique appliquée et par la psycholinguistique cognitive nous expliquent qu'en L2 plusieurs procédés et facteurs linguistico-cognitifs interagissent dans l'accès lexical en passant de la phase de compréhension à celle de la production. Dans notre étude, nous essayons de prendre en compte très brièvement quelques-uns de ces procédés et facteurs, indépendamment du fait qu'ils soient réalisés volontairement ou involontairement par les apprenants en phase productive.

Les procédures cognitives qui se déroulent dans l'accès lexical ont recours autant à la mémoire lexicale qu'aux compétences métalinguistiques de l'apprenant. Notamment, d'après Bogaards, le degré de difficulté d'un mot n'est pas inscrit dans ses propriétés intrinsèques mais plutôt dans les relations entre les 
items lexicaux et les apprenants. Ces relations peuvent être résumées en trois points (Bogaards, 1994 : $151-153)$ :

- le traitement contrastif des mots («pour être mieux encodé et plus facilement retrouvable, tout item lexical a besoin d'être traité avec assez d'attention ; c'est grâce à la contrastivité du traitement que se crée ou s'affermit sa représentation mentale »- Bogaards, $1994: 153$ );

- la familiarité (les mots familiers en L2 seront reconnus plus facilement que les autres; les mots les plus difficiles sont ceux dont le sens n'est pas codé dans les concepts du réseau sémantique de la L1);

- les mots concrets (ils peuvent être reconnus et mémorisés plus facilement car l'apprenant peut les associer à des images bien précises).

Nous allons illustrer comment ces trois facteurs peuvent avoir influencé l'encodage lexical en production écrite.

\subsection{Le rôle du traitement contrastif et en profondeur des mots : connaissances lexicales et coïncidences morphosémantiques parmi les langues connues}

Nous avons affirmé qu'une erreur peut être analysée comme étant le résultat de plusieurs procédés cognitifs. Un exemple éclairant est la cible *surprisé pour surpris (participe passé) dans «l'ami de la fille a été *surprisé [...]». Cette erreur est probablement due au niveau d'interlangue en LC de l'étudiant : il s'agirait d'une confusion/substitution lexicale entre le V ou l'ADJ surpris et le $\mathrm{N}$ (une) surprise, ou bien d'une surgénéralisation de la marque du participe passé des verbes en -er. Nous pouvons également interpréter cette occurrence comme une interférence interlinguistique, ou un calque, notamment (1) depuis l'italien, il s'agissait sans doute d'un calque de l'it. sorpresa (N, « (une) surprise »; ADJ, « (être) surprise », et participe passé du V sorprendere, «surprendre ») et (2) depuis l'anglais surprised (ADJ, «(être) $\operatorname{surpris}(\mathrm{e}) »)$.

La performance dans l'encodage d'items est le plus souvent reliée à la compétence lexicale de l'étudiant, à son niveau de connaissance des mots, notamment lorsqu'il faut produire des formes complexes et non transparentes en LM (dans notre étude, l'arabe). Il arrive souvent qu'un phénomène en apparence interférentiel soit en réalité le résultat de l'état de l'apprentissage lexical et morphosémantique de l'apprenant, le reflet de sa propre interlangue (Colombo, 2009 : 123). Toutefois, les rédacteurs pourraient agir par analogie en reproduisant des items-modèles enregistrés en mémoire, ce qui témoignerait d'une réflexion métalinguistique et d'une analyse intralinguistique effectuées en profondeur (Colombo \& Tea, 2011) : il s'agirait de ce que Frei appelle l'assimilation mémorielle, c'est-à-dire la modification ou la création d'un élément linguistique par imitation d'un autre modèle linguistique (Cuq, 1996: 47-48). Selon l'hypothèse dite de la profondeur de codification ou Levels of Processing (Niveaux de Traitement) de Craick \& Lockart (1972), les informations traitées sont d'autant mieux retenues et susceptibles d'être retrouvées dans la mémoire sémantique qu'elles sont traitées en profondeur: est considéré comme superficiel un traitement qui se concentre sur la forme, même si celle-ci s'accompagne d'une répétition dans l'apprentissage; en revanche, un traitement en profondeur sur le sens permettra à ce dernier de devenir plus prégnant et plus accessible dans la mémoire lexicale :

C'est la tâche à exécuter qui impose le niveau de traitement: ainsi, la simple répétition d'un élément n'implique que des niveaux superficiels, tandis que les tâches difficiles comme celles qui font appel à des facultés de raisonnement ou à la comparaison détaillée, et qui font donc intervenir le sens du matériel utilisé, ont lieu à un niveau profond (Bogaards, 1994 : 91).

La reproduction d'un item lexical par analogie ferait partie d'un ensemble plus vaste de compensatory strategies (Kellerman, 1991), ou des stratégies compensatoires (Gaonac'h \& Merlet, 1995), mises en œuvre par l'apprenant et consistant soit à combler le vide d'un item dans son dictionnaire lexical, soit à combler le manque d'efficacité dans l'accomplissement des tâches en entrée lexicale : 
In Faerch \& Kasper's (1983) taxonomy of strategies, Nigel Barley's'hot beasts' would be a token of a subset of communication strategy types known compensatory strategies, since these are used to maintain the integrity of the learner's original communicative goal. Specifically they are lexical compensatory strategies, since they replace missing lexical items (Kellerman, 1991 : 143).

Parmi ces stratégies, nous comptons également l'exploitation des coïncidences entre la LM, les autres L2 connues par l'apprenant et la langue cible (LC), c'est-à-dire le français. De ce point de vue, Bardel constate :

[qu']alors que l'apprenant, en acquérant une L2, ne peut bénéficier que de son expérience d'apprentissage de la [L1], l'apprenant d'une L3 a déjà l'expérience de l'apprentissage d'une ou de plusieurs autres langues étrangères, ce qui, à son tour, constitue un avantage en ce qui concerne les stratégies et les connaissances métalinguistiques (Hufeisen, 1998: 171). [La] L3 peut influencer la L1 et subir l'influence (L1-L3), [et] des influences linguistiques réciproques peuvent s'installer entre la L2 et la L3 (Bardel, 2006 : 150).

Plus précisément, il nous semble que nos étudiants ont souvent eu recours à d'autres L2 différentes du FLE, au moyen de transferts interlinguistiques positifs et négatifs, en entendant par transfert interlinguistique le «passage automatique de la LM [ou d'une autre L2] qui est déterminé par la structure des langues en contact» (Giacobbe, 1990 ; cité par Masperi, 1998 : 177). Il s'agit ici d'interférences lexicales (graphophonologiques ou encore morphosémantiques, particulièrement des calques) ou structurelles (syntaxiques) d'autres L2 connues, notamment l'italien et l'anglais. Voici quelques exemples :

\section{(A) Interférences et calques depuis l'anglais}

- des interférences lexicales : la réalisation *problem pour problème est sans doute le résultat d'une interférence graphophonologique depuis l'ang., voire un calque total depuis cette LE; (un homme) *masculé pour (un homme) musclé, calqué sur l'ang. muscular (" (un corps) musclé »); le *privet pour le privé (dans le sens de « la vie privée), un calque phonétique de l'ang. private («privé »); *carecter pour personnage, un calque graphique et total de l'ang. caracter («personnage »);

- des calques structurels : * sur la terre/*en terre pour par terre, de l'ang. on earth / on the floor / $\underline{\text { on }}$ the ground («sur terre, par terre, sur le sol»); il/l'homme *a tombé amoureux pour il/l'homme est tombé amoureux, de l'ang. the man has fallen («l'homme est tombé ») et he has fallen in love (« il est tombé amoureux »); on *a sorti pour on est sorti, de l'ang. we have left (« nous sommes sortis ; on est sorti »).

\section{(B) Interférences et calques depuis l'italien}

- des interférences lexicales: la correspondance graphie/phonie de l'it. tempo ['tempo] peut avoir interféré dans l'encodage du fr. *tempe [temp] pour temps [tã]; le graphème $<\mathrm{u}>$ qui attaque l'it. uomo ['wo:mo] peut avoir interféré dans l'encodage de la première voyelle du fr. *humme pour homme ;

- des calques structurels: * $\underline{\text { le }}$ météo pour la météo est peut-être calqué sur le genre masculin de l'it. il meteo (« la météo »); (la femme) * non *accepete pour (la femme) n'accepte pas, calqué totalement sur la forme négative italienne non $+V$ (par exemple, la donna non accetta, « la femme n'accepte pas »).

D'après la théorie du processus de procéduralisation (Bange, 2006 : 58-62), au fur et à mesure que l'apprentissage d'une L2 progresse, le lien L1/L2 aurait tendance à réduire son emprise : le transfert translinguistique ne serait qu'une des opérations cognitives pouvant intervenir dans l'accès au lexique. Il semblerait que « les transferts sont plus évidents en production qu'en compréhension où ils se produisent pourtant de la même manière » (Jamet, 2009 : 55). Notamment, en ce qui concerne les interférences, elles peuvent comporter des aspects positifs dans les pratiques d'apprentissage/enseignement d'une L2. En effet, du côté du formateur, l'interférence lui permettrait d'évaluer la progression en L2 de la part de l'étudiant par rapport aux objectifs visés (Cuq, 1996 : 50-51); du côté de l'apprenant, selon Degache \& Masperi (1998), le nombre des transferts négatifs est généralement inférieur à celui des transferts positifs, ce qui impliquerait qu'il serait raisonnable de s'appuyer sur les similitudes morphosémantiques entre 
deux et plusieurs langues (sœurs) dans l'apprentissage d'une L2. Étant donné également «[qu']il n'est désormais plus nécessaire d'argumenter l'existence même d'une influence du facteur translinguistique dans l'apprentissage d'une L2 » (Ó Laoire \& Singleton, 2006: 102), l'exploitation de la proximité interlinguistique et des compétences métalinguistiques des apprenants doit être privilégiée, d'après nous, dans l'apprentissage d'une ou de plusieurs L2 : la proximité entre la LC et l'italien L2 aurait permis des transferts positifs parmi ces deux langues sœurs, ce qui impliquerait que les apprenants aient effectué une analyse contrastive et une réflexion métalinguistique approfondies. Le transfert positif est d'autant plus probable que les conditions du transfert sont respectées : à ce sujet, l'approche psychotypologique d'Eric Kellerman (1977-1980) cite la nécessité d'une «évaluation subjective [positive] de la distance typologique entre les langues » (Rast, $2006: 120$ ) ; le transfert de la L1 à la L2 est une stratégie mise en œuvre par l'apprenant, une sorte d'interaction perpétuelle entre l'étudiant et ses facteurs psychologiques et linguistiques. Selon Kellerman, le transfert d'une langue à l'autre aura plus de chances d'être réalisé si les contraintes sur le transfert (positif) sont respectées (Masperi, 1998 : 182-183). Dans les productions écrites observées, les transferts positifs de l'italien au français concerneraient autant des mots pleins que des mots vides (grammaticaux) :

- coïncidences morphosémantiques totales it./fr. : it. grande/fr. grande, tu/tu, un/un, lui/lui, meteo/météo, etc. ;

- coïncidences morphosémantiques partielles it./fr.: it. decidere/fr. décider, fortemente/fortement, terra/terre, pensare/penser, portare/porter, amore/amour, finire/finir, forte/fort ; etc.

Nous ne sommes pas en mesure d'affirmer que l'espagnol L3/Ln est intervenu dans l'activation lexicale en français, au moyen de transferts morphosémantiques, étant donné que seuls deux des 10 rédacteurs ont appris cette L2, et ceci seulement pendant une année de formation universitaire. Ces constats nous empêchent de formuler et de généraliser des hypothèses à l'égard de transferts potentiels parmi ces deux langues romanes.

Nous concluons en affirmant que lorsqu'un item en LC ne fait pas partie du dictionnaire de l'apprenant, ou n'est pas retrouvé dans la mémoire à long terme de l'étudiant, le sujet peut recourir à plusieurs procédés conscients ou inconscients, de nature interlinguistique (si la distance entre les langues est perçue positivement) ou intralinguistique, pouvant ainsi répondre à ses besoins communicatifs. En effet, en présence d'un vide lexical, l'étudiant se trouve généralement face à un choix stratégique : soit laisser un vide dans sa production, soit avoir recours à des stratégies compensatoires dans l'intention de faire passer son message, même si le résultat est parfois négatif, comme dans le cas des interférences et des substitutions paradigmatiques (néanmoins, l'échange lexical permet au sujet de se positionner dans un champ lexico-sémantique pertinent vis-à-vis de l'item-cible). Plus généralement, Pichiassi (1999: 255) précise que les stratégies compensatoires sont mises en action par l'apprenant pour compenser des incompréhensions, ce qui est dû à une connaissance limitée de la L2; et que d'après Cattana \& Nesci (2004 : 96) lorsque l'apprenant ne connaît pas une expression de la L2, les stratégies de compensation ou de communication lui permettent, souvent, au moins de se faire comprendre. Pour finir, Mezzadri (2003 : 37) cite Oxford lorsque ce dernier considère que les stratégies d'apprentissage, qui sont souvent, mais pas toujours, intentionnelles, sont des actions ou des techniques utilisées dans l'objectif d'améliorer la progression des habilités en L2, en facilitant l'intériorisation, le stockage lexical, la récupération et l'utilisation de nouvelles connaissances linguistiques.

\subsection{Le rôle de la fréquence et de la familiarité lexicales}

La fréquence, c'est-à-dire «le nombre de fois où un même mot revient dans un corpus (oral ou écrit) » (Babin, 1998:20), a une influence directe sur le temps nécessaire à la reconnaissance lexicale, sur la prononciation, la compréhension, l'association, etc. : plus un mot est objectivement fréquent dans une langue donnée, plus la probabilité de le rencontrer est élevée pour un locuteur. Les mots objectivement les plus rencontrés et utilisés (fréquents) sont aussi les plus familiers du point de vue subjectif: par familiarité des mots, nous entendons ici une variable psychologique correspondant aux expériences subjectives, en réception et en production, qu'un individu a eues avec les mots. Chaque rencontre 
provoque un traitement perceptif et sémantique, ce qui laisse une trace en mémoire ; cela s'accumule sur la représentation de la forme du mot dans le lexique mental, ce qui revient à lui donner un air de familiarité. En règle générale, plus un mot est fréquent et plus le temps d'identification est court. Selon la loi de l'exercice (Anderson, 1983; cité par Babin, 1998: 21-22), les traitements lexicaux sont susceptibles de voir leur vitesse et leur exactitude augmenter avec le nombre de leurs répétitions : «les mots qui sont souvent rencontrés dans le discours, sont donc souvent traités par le système lexical. [...] Cet effet [semblerait] relativement universel » (ibidem). Ainsi, il est important que les apprenants d'une L2 puissent entrer en contact plusieurs fois avec le même input lexical.

Les lemmes les plus fréquemment cités dans les productions écrites que nous avons analysées sont (en ordre décroissant de récurrence) les noms homme(s) (30 occurrences), fille et femme (15 occurrences chacun) ; suivent le verbe frapper (frapper, frappé(e) : 9), amour/aimer/amoureux (7), journal et grand (6), l'adjectif fâché(e) (5), les noms mari/(la) marié et amis (4 occurrences chacun), garçon (3), et ainsi de suite. La plupart de ces mots sont d'ailleurs signalés par la majorité des corpus du français parlé existants et accessibles en ligne. Bien que l'observation des corpus du français (oral et écrit) n'entre pas dans le cadre de notre étude, nous citons, à titre d'illustration, le corpus hébergé par la plate-forme CLAPI (Corpus de Langues Parlées en Interaction) : il s'agit d'un corpus facilement accessible en ligne (à l'adresse : http ://clapi.univ-lyon2.fr/V3_Accueil.php) et résultant de recherches individuelles et collectives, notamment de plusieurs équipes de recherche parmi lesquelles UMR-ICAR ${ }^{4}$. Parmi les 500 mots les plus fréquents signalés par CLAPI, nous trouvons les lemmes garçon (position 125/500), aimé (237/500), fille (309/500), belle (363/500), grand/grande/grands (respectivement en position 350, 354 et $471)$, et fort (384/500) : tous ces mots ont été employés par nos étudiants. Ce résultat est encore plus réconfortant si l'on considère que parmi les 500 mots les plus fréquents figurant dans CLAPI, les mots vides occupent une place importante ${ }^{5}$. D'ailleurs, selon Zagrebelsky (1998) les mots fonctionnels sont les plus fréquents dans une langue donnée. Indépendamment de ce que les corpus de la langue française nous disent à propos de la fréquence des mots, et du fait que ces corpus soient (comme nous l'espérons) ou non en liaison avec les activités effectuées en classe de langue, les mots cités sont également fréquemment rencontrés dans les cours de FLE : des couples antonymiques tels que hommel femme ou mari/femme, garçon/fille, petit/grand, des lemmes pour désigner les expressions et les relations affectives (ami(e), amour, aimer, amoureux) sont vraisemblablement reçus, traités et employés dans des cours de langue étrangère.

\subsection{Le rôle de la perception de l'image et du trait sémantique [+concret]}

Selon Balboni (1994 : 37-38) les processus mis en action pendant une production sont la définition de la typologie du texte à produire (dans notre cas, narratif), le plan qui influencera le contenu du texte, la rédaction d'un texte correct et homogène et en dernier lieu l'analyse du contexte situationnel. Dans le cadre d'une traduction intersémiotique image/langage verbal, la perception de l'image (par exemple, l'image VALICO) stimulerait une analyse visuelle et une exploitation des repères contextuels (situationnels). Le résultat est une attention sélective non négligeable ou une focalisation sur les référents prioritaires (les personnages; quelques objets) au détriment d'autres référents. Les vignettes LOVE mettent au premier plan des référents immédiats et prioritaires de l'image : les deux hommes et la femme. Ces référents sont perceptivement prioritaires étant donné (a) qu'ils occupent l'espace le plus imposant dans l'illustration; (b) qu'ils sont dessinés selon des symétries presque parfaites, de gauche à droite, de haut en bas, ce qui invite le compreneur ${ }^{6}$ à valoriser simultanément la symétrie de ces référents ; $(c)$ l'amorce proposée ( «hier au parc...») constitue un renfort cognitif dans cette direction, car elle stimule une attention sélective vers les personnages porteurs de l'action. Dans le dessin d'entrée, la première vignette, les trois personnages sont positionnés au premier plan, ce qui leur permet d'obtenir une attention particulière : l'attention sélective sur ces référents peut être considérée ainsi comme l'une des variables qui expliquent la récurrence des mots homme(s), femme et fille dans les productions et, par conséquence, des mots associés à ces personnages (des noms - journal, mari, force, amour -, des adjectifs - faché(e), amoureux, grand, fort-, des verbes - frapper, porter -, etc.) comme, d'ailleurs, des structures syntaxiques presque obligatoires en vue de la situation à décrire (être/tomber amoureux de). Comme nous pouvons le 
remarquer, les référents prioritaires des vignettes sont désignés par des noms caractérisés par le trait sémantique $[+$ concret $]$ : le caractère concret du nom permettrait aux sujets de se représenter objectivement le référent et, par conséquent, de le rendre cognitivement plus accessible et utilisable en production (écrite). Nos observations concordent aussi bien avec le discours de Boogards (que nous avons exposé plus haut) à l'égard de la représentation cognitive référent/désignateur concret, qu'avec la théorie du double code d'Alain Paivio (1971; cité par Cardona, 2004 : 156) selon qui si le langage verbal favorise le stockage des mots abstraits et concrets dans la mémoire à long terme, la visualisation dans des images ne permettrait que le stockage des mots concrets car ils peuvent être facilement représentés.

Selon notre hypothèse, lorsqu'en phase de compréhension de l'image les sujets perçoivent les référents immédiats, ils leur associent un sens précis : les étudiants effectueraient l'abstraction de sens autour des désignateurs linguistiques correspondants. L'opération suivante correspondrait à l'assemblage des signifiés par synthèse sémantique, c'est-à-dire une activité cognitive où « [...] l'esprit / cerveau d'un locuteur, mis en présence d'un énoncé, lie mentalement deux représentations » (le Ny, $2005: 358$ ). Le résultat correspond à la naissance du sens à exprimer (ibidem: 100), c'est-à-dire à une intention sémantique de dire quelque chose (ibidem). Une fois le sens initial mis en place, il donnerait vie au sens terminal à exprimer quelque chose qui se positionne "à l'autre bout du schéma de communication cognitive, au produit de la compréhension » (ibidem: 101). C'est la phase de production écrite : les étudiants emploieraient soit des mots types retrouvés en mémoire, soit des structures syntaxiques. Indépendamment du schéma suivi, les sujets analyseraient le contexte pour accomplir la tâche productive. Par conséquent, pour l'accomplissement d'opérations de bas niveau (notamment, d'accès au lexique), les sujets seraient incités à donner la priorité à la désignation et à la qualification des référents cibles.

\section{CONCLUSIONS}

Nous avons affirmé combien l'exploitation de la proximité interlinguistique et des compétences métacognitives (à propos de la perception de l'image, de la fréquence et de la familiarité du lexique, etc.) est importante dans la classe de langue. La principale conclusion que nous proposerons selon notre expérience et les résultats de notre analyse est que dans l'enseignement d'une langue étrangère, la connexion avec la culture qui y est associée, liée même, est fondamentale. La communication entre apprenant et natif reste très souvent incompréhensible si on ne tient pas compte du trait culturel ou du contexte socioculturel. Le concept de «lexiculture» devrait donc être appliqué prioritairement dans l'apprentissage / enseignement des langues-cultures et se trouver au cœur de la didactique des L2. La lexiculture est présentée comme une méthode qui utilise un lexique culturel spécifique à la langue enseignée. Peut-être faut-il élargir encore l'enseignement simultané de la culture pour «baigner » les apprenants dans l'univers de la langue L2 car les effets positifs et dynamiques sont très nombreux. Outre la compréhension de la langue, cette forme d'enseignement crée aussi, une ouverture vers les autres. Elle introduit dans la classe un monde extérieur avec ses dimensions socioculturelles ou psycholinguistiques. L'apprenant découvre en même temps la langue concrètement, pratiquement et sera beaucoup plus intéressé, curieux et motivé. Dans la progression de l'apprentissage, les phases de nativisation et d'interlangue pourront être écourtées car l'apprenant sera amené à faire des comparaisons entre les langues et leurs cultures plutôt que des substitutions. Il ne saurait être question, en effet, de substituer la culture de l'apprenant qui est et sera toujours présente. Elle est une base pour une relativisation et un élargissement vers le contexte enseigné afin de l'intégrer mieux et plus vite.

\section{Références bibliographiques}

Andersen, R. (1983). Pidginization and Creolization in Language Acquisition. Rowley, MA: Newbury House.

Anderson, J. (1993). Rules of the Mind. Hillsdale, NJ: Lawrence Erlbaum. 
Babin, J. P. (1998. Lexique mental et morphologie lexicale, Sciences pour la Communication n. 54, Bern-BerlinFrankfurt-New York-Paris-Wien : Peter Lang SA-Ed. Scientifiques Européennes.

Balboni, P. (1994). Didattica dell'italiano a stranieri. Roma : Bonacci.

Bange, P. (2006). Les conditions internes et externes de l'apprentissage des langues étrangères. In : Faraco, M. (Éd.), La classe de langue, théories, méthodes et pratiques. Aix-en-Provence: Publications de l'Université de Provence, p. 47-70.

Barbera, M., Marello, C. (2004). VALICO (Varietà di Apprendimento della Lingua Italiana Corpus Online) : una presentazione. In: Blaboni, P. E., Docli, R., Serragiotto, G. (Éd.), ITALS-Italiano Lingua Straniera Dipartimento di Scienze del Linguaggio, année II, n. 4, p. 7-18.

Bardel, C. (2006). La connaissance d'une langue étrangère romane favorise-t-elle l'acquisition d'une autre langue romane? Influences translinguistiques dans la syntaxe en L3. In : Rast, R., Trévisol, P. (Éd.), L'acquisition d'une langue 3, Acquisition et Interaction en Langue Étrangère (AILE), n. 24. Paris : Encrages, 149-179.

Bogaards, P. (1988). Aptitude et affectivité dans l'apprentissage des langues étrangères. Paris : Hetier.

Calaque, É. (2000). Enseignement et apprentissage du vocabulaire. Hypothèses de travail et propositions didactiques. In : Calaque, E., Grossmann, F. (Éd.), Enseignement / apprentissage du lexique, Lidil, n. 21. Grenoble : EllugUniversité Stendhal, p. 17-36.

Cardona, M. (2004). Apprendere il lessico di una lingua straniera. Aspetti linguistici, psicolinguistici e glottodidattici. Bari : Adriatica Editrice.

Cattana, A., Nesci, M. T. (2004). Analizzare e correggere gli errori. Collection : Marello, C. (Éd.), L'officina della lingua. Formazione Insegnanti Italiano Lingua straniera. Perugia : Guerra.

Charaudeau, P. (1987). L'interculturel: nouvelle mode ou pratique nouvelle, Le Français dans le Monde, Recherches et Applications, $\mathrm{n}^{\mathrm{0}}$ spécial février-mars, p. 25-33.

Coirier, P., Gaonac'h, D., Passerault, J. M. (1996). Psycholinguistique textuelle : une approche cognitive de la compréhension et de la production des textes. Paris : Armand Collin.

Colombo, O., Tea, E. (2011). Les compétences plurilingues et métalinguistiques lors de la compréhension et la production écrites des noms dérivés en italien LE dans un forum en ligne. EPAL (Échanger pour Apprendre en Ligne). Colloque International, Université Stendhal-Grenoble 3, 23-25 juin. Accessible à l'adresse http://w3.ugrenoble3.fr/epal/dossier/06_act/actes2011.htm.

Colombo, O. (2009). L'apprendimento dell'alterazione in italiano L2 in produzioni scritte di francofoni. In : Corino, E., Marello, M. (Éd.), VALICO. Studi di linguistica e didattica, Perugia, Guerra Edizioni, p. 111-135.

Conseil de l'Europe. (2001). Cadre européen commun de référence pour les langues: Apprendre, Enseigner, Évaluer. Paris : Didier.

Corda, A., Marello, C. (2004). Lessico insegnarlo e impararlo. Perugia : Guerra Edizioni.

Coste, D. (1975). Les piétinements de l'image. In : Coste, D. (Éd.), Image en didactique des langues, Études de linguistique appliquée, n. 17-1975. Paris : Didier, p. 5-28.

Cuq, J. P. (1996). Une introduction à la didactique de la grammaire en français langue étrangère. Paris : Didier / Hatier.

Degache, C., Masperi, M. (1998). La communication plurilingue en toile de fond de l'entraînement à la compréhension des langues romanes. In : Billiez, J. (Éd.), De la didactique des langues à la didactique du plurilinguisme, hommage à Louise Dabène. Grenoble: Lidilem-Université Stendhal, p. 361-376. www.ugrenoble3.fr/galatea/dcmm1998.htm, www.galanet.eu/publication/fichiers/dc-mm1998.pdf.

Demaizière, F., Narcy-Combes, J.P. (2005). Méthodologie de la recherche didactique : nativisation, tâches et TIC, Alsic, vol. 8, $\mathrm{n}^{\circ} 1$ | 2005. http://alsic.revues.org/326 ; DOI : 10.4000/alsic.326

Duquette, L., Tréville, M.C. (1996). Enseigner le vocabulaire en classe de langue. Paris : Hachette.

Eco, U. (1996). Interprétation et surinterprétation. Paris : Presses Universitaires de France. Traduction de Cometti, J.P., de l'ouvrage : 1992, Interpretation and overinterpretation. Cambridge : Cambridge University Press. 
Ferreri, S. (2005). L'estensione delle conoscenze lessicali individuali. In : Chiari, I., De Mauro, T. (Éd.), Parole e numeri. Analisi quantitative dei fatti di lingua. Roma: Aracne, p. 307-334.

Galisson, R. (1988). Culture et lexiculture partagées : les mots comme lieux d'observation des faits culturels, Études de Linguistique Appliquée, n. 69, p. 74-90.

Galisson, R. (1986). Éloge de la "Didactologie/Didactique des langues et des cultures (Maternelles et étrangères)", —D/DLC—》, Études de Linguistique Appliquée, n. 64, p. 38-54.

Galisson, R. (1984). Dictionnaire de compréhension et de production des expressions imagées. Paris: Clé International.

Galisson, R. (1983). Image et usage du dictionnaire chez des étudiants (en langue) de niveau avancé», Études de Linguistique Appliquée, n. 49, p. 5-88.

Gaonac'h, D., Merlet, S., (1995). Mise en évidence de stratégies compensatoires dans la compréhension orale en L.E. Revue de phonétique appliquée, n. 115-116-117, Compréhension orale. Mons : Université de Mons-Hainaut, p. 273-292.

Jamet, M. C. (2009). Contacts entre langues apparentées : les transferts négatifs et positifs d'apprenants italophones en français. In : Raus, R. (Éd.), Rencontre des langues et politique linguistique, Synergies Italie, n. 5. Sylvain les Moulins : Gerflint, 49-59.

Kellerman, E., (1991). Compensatory strategies in second language research: a critique, a revision, and some (non-) implications for the classroom. In : Phillipson, R., Kellerman, E. \& al. (Éd.), Foreign / Second Language Pedagogy Research. Multilingual Matters : Clevedon, p. 142-161.

Le Ny, J. F. (2005). Comment l'esprit produit du sens. Paris : Odile Jacob.

Mallet, B. (1989). Réperages. Image en mouvement et contexte pédagogique. Le rapport du filmique et du scriptural. In : Dabène, M. (Éd.), Regards sur la lecture : textes et images. Grenoble : Ellug, p. 119-136.

Masperi, M. (1998). Regards sur la proximité typolinguistique dans la perspective d'une approche contrastive rénovée. In: Masperi, M., Étude exploratoire des conditions d'autonomisation de lecteurs francophones débutants en italien, Thèse de Doctorat Université Grenoble III. Villeneuve d'Ascq : Presses Universitaires du Septentrion, chapitre 2, p. 176-196.

Masperi, M. (1996). Quelques réflexions autour du rôle de la parenté linguistique dans une approche de la compréhension écrite de l'italien par des francophones débutants. In : Dabène \& L., Degache, C. (Éd.), Comprendre les langues voisines. Etudes de Linguistique Appliquée, n. 104, octobre-décembre 1996. Paris: Didier-Erudition, chapitre onzième, p. 491-502.

Meara, P. (1980). Vocabulary acquisition: a neglected aspect of language learning. Language teachng \& linguistics abstracts, 13,4 , p. 221-246.

Mezzadri, M. (2003). I ferri del mestiere. (Auto)formazione per l'insegnante di lingue. Perugia : Guerra Edizioni.

Ó Laoire, M., Singleton, D. (2006). Psychotypologie et facteur L2 dans l'influence translexicale. Une analyse de l'influence de l'anglais et de l'irlandais sur le français L3 de l'apprenant. In: Rast, R., Trévisol, P. (Éd.), L'acquisition d'une langue 3, Acquisition et Interaction en Langue Étrangère (AILE), n. 24. Paris : Encrages, p. 101-117.

Petiot, G., Reboul-Touré, S. (2005). Apprentissage et enseignement du lexique : pour une didactique mettant en œuvre les discours et la langue. In : Grossmann, F., Paveau, M. A., Petit, G. (Éd.), Didactique du lexique : langue, cognition, discours. Grenoble : ELLUG-Université Stendhal-Grenoble 3, p. 215-226.

Pichiassi, M., Zaganelli, G. (1992). Contesti Italiani. Materiali per la didattica dell'italiano L2. Viaggio nell'italiano contemporaneo attraverso i testi. Perugia : Guerra Edizioni.

Polianov, E. (1931). La perception des sons d'une langue étrangère. In : Travaux du Cercle linguistique de Prague, $\mathrm{n}$. 4, p. 79-96.

Rast, R. (2006). Le premier contact avec une nouvelle langue étrangère : comment s'acquitter d'une tâche de compréhension ?. In : Rast, R. \& Trévisol, P. (Éd.), L'acquisition d'une langue 3, Acquisition et Interaction en Langue Étrangère (AILE), n. 24. Paris : Encrages, p. 119-147. 
Tardy, M. (1975). La fonction sémantique de l'image. In : Coste, D. (Éd.), Image en didactique des langues, Études de linguistique appliquée, n. 17-1975. Paris : Didier, p. 29-43.

Véronique, G. D. (2007). L'action en classe de langues et les activités de recherche en didactique des langues et des cultures, Cahier de l'Acedle, n. 4, p. 121-132. Accessible à l'adresse http://acedle.org/IMG/pdf/Veronique_cah4.pdf

Widdowson, H. (1978). Teachering language as communication. Oxford : Oxford University Press. 


\section{ANNEXE 1 : vignettes $L O V E$}

Guarda queste figure e scrivi una storia: non devi descrivere i disegni uno per uno, perché anche una persona che non vede le figure deve capire cosa è successo.

Usa più di 100 parole e inizia così: Ieri al parco... (Hier aux jardins publics...)
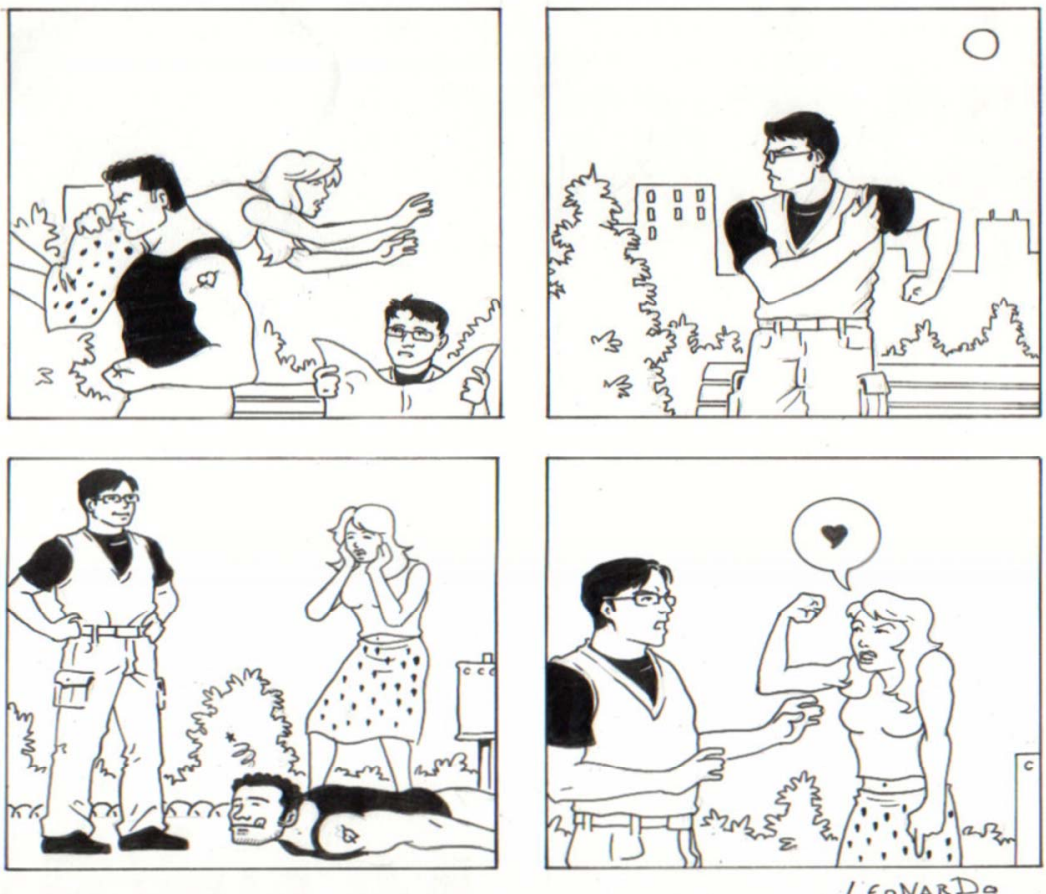

\footnotetext{
${ }^{1}$ Le projet est né en 2003 à l'Université de Turin (Italie) grâce au travail de Carla Marello, Elisa Corino et Manuel Barbera entre autres. Pour plus d'informations, voir : Barbera, M., Marello, C. (2004).

${ }^{2}$ Les vignettes LOVE sont en Annexe 1.

${ }^{3}$ L'expérience a été menée pendant le second semestre de l'année universitaire 2011/2012.

${ }^{4}$ Pour plus d'explications sur la création du corpus CLAPI : http://icar.univ-lyon2.fr/projets/corinte/bandeau_gauche/clapi.htm.

${ }^{5}$ Par exemple, parmi les 100 mots les plus fréquents qui figurent dans le corpus CLAPI, nous avons enregistré 72 mots vides et seulement 28 mots pleins au total.

${ }^{6}$ Selon Le Ny (2005), un compreneur est une personne qui se trouve face à une tâche de compréhension orale ou écrite.
} 